\title{
Giant Cell Arteritis among Fevers of Unknown Origin (FUO): An Atypical Presentation
}

\author{
Lorenzo Grazioli-Gauthier ${ }^{1}$, Natalie Marcoli ${ }^{1,2}$, Gianluca Vanini $^{1,3}$, Enos Bernasconi ${ }^{1,4}$, Dea Degabriel $^{1}$ \\ ${ }^{1}$ Department of Internal Medicine, Ospedale Regionale di Lugano, Ente Ospedaliero Cantonale, Lugano, Switzerland \\ ${ }^{2}$ Department of Rheumatology, Ospedale Regionale di Lugano, Ente Ospedaliero Cantonale, Lugano, Switzerland \\ ${ }^{3}$ Department of Immunology and Allergology, Ospedale Regionale di Lugano, Ente Ospedaliero Cantonale, Lugano, Switzerland \\ ${ }^{4}$ Department of Infectious Diseases, Ospedale Regionale di Lugano, Ente Ospedaliero Cantonale, Lugano, Switzerland
}

Received: 29/12/2020

Accepted: 26/01/2021

Published: 09/02/2021

How to cite this article: Grazioli-Gauthier L. Marcoli N. Vanini G, Bernasconi E, Degabriel D. Giant cell arteritis among fevers of unknown origin (FUO): an atipical presentation. EJCRIM 2021;8: doi:10.12890/2021_002254.

Conflicts of Interests: The Authors declare that there are no competing interests.

This article is licensed under a Commons Attribution Non-Commercial 4.0 License

\section{ABSTRACT}

Giant cell arteritis (GCA), or Horton's arteritis, presenting solely as fever is very rare. Usually, it manifests with typical features such as visual problems, headache and jaw claudication, or it can be associated with polymyalgia rheumatica. We describe the case of a patient with GCA who presented only with prolonged fever, the cause of which was not determined by diagnostic tests.

\section{LEARNING POINTS}

- Fever may be the only symptom of giant cell arteritis (GCA).

- It is important to consider GCA in the differential diagnosis of fever of unknown origin as early diagnosis is crucial for prompt treatment and to prevent catastrophic complications such as vision loss or stroke.

- Temporal artery biopsy remains the gold standard for diagnosing GCA.

\section{KEYWORDS}

Fever of unknown origin (FUO), vasculitis, giant cell arteritis (GCA), Horton's arteritis

\section{CASE DESCRIPTION}

A 75-year-old man was admitted to the internal medicine department for clinical investigation of fever $\left(38^{\circ} \mathrm{C}\right)$. The patient reported a 3 -day history of low-grade fever and muscle pain mainly in the lower limbs. His general practitioner had performed laboratory tests which showed systemic inflammation with C-reactive protein (CRP) $196 \mathrm{mg} / \mathrm{I}$ (n.v. $<5 \mathrm{mg} / \mathrm{I}$ ) and leucocytosis with neutrophilia 12.6×10\%/I (n.v. 4-10×10\%/I). The patient denied any accompanying symptoms such as cough, diarrhoea, abdominal pain, dysuria, headache or pharyngitis. He did not have contact with anyone known to be positive for COVID-19. He had not travelled abroad recently, was not currently taking any medication and did not smoke.

On admission, the patient was in good physical condition. His blood pressure (BP) was 119/72 mmHg, heart rate (HR) was 73 bpm, body mass index (BMI) was $26 \mathrm{~kg} / \mathrm{m}^{2}$ and his temperature was elevated at $38^{\circ} \mathrm{C}$. Oxygen saturation on room air was $96 \%$. The physical examination was normal. Repeat laboratory tests demonstrated an increase in systemic inflammation with CRP at $250 \mathrm{mg} / \mathrm{l}$ with high procalcitonin (PCT) of $0.79 \mu \mathrm{g} / \mathrm{l}$ (n.v. $<0.25 \mu \mathrm{g} / \mathrm{l}$ ) and leucocytosis with neutrophilia at $12.5 \times 10^{9} / \mathrm{l}$ (n.v. $4-10 \times 10^{9} / \mathrm{I}$ ) as well as an increase in erythrocyte sedimentation rate (ESR) to $70 \mathrm{~mm} /$ hour (n.v. $<10 \mathrm{~mm} /$ hour). 
During the first 5 days of hospitalization, we observed persistent fever $\left(38-38.5^{\circ} \mathrm{C}\right)$ and worsening systemic inflammation with a rise in CRP to $367 \mathrm{mg} / \mathrm{l}$ and in leucocytosis with neutrophilia to $15.5 \times 10^{\%} / \mathrm{I}$. There was also mild anaemia with Hg $122 \mathrm{~g} / \mathrm{l}$ (n.v. 140-180 g/l) and thrombocytosis up to $940 \times 10^{9} /$ I (n.v. $150-400 \times 10^{\%} /$ I). The haematological findings on peripheral smear confirmed neutrophilic leucocytosis and eosinophilia, monocytes and reactive lymphocytes as well as mild normocytic normochromic anaemia and thrombocytosis, interpreted as reactive to the ongoing inflammatory process. Serum protein electrophoresis showed hypoalbuminemia and increased alpha fractions compatible with possible acute infection or malignancy. The serological panel for bacterial and viral infections (human immunodeficiency virus, viral hepatitis A, B, C, Bartonella henselae and B. quintana, Toxoplasma gondii, Coxiella burnetii, Chlamydia trachomatis, Tropheryma whipplei PCR, Treponema pallidum screening) excluded ongoing infection. Autoimmune screening was negative for antinuclear antibody (ANA), antineutrophil cytoplasmic antibody (ANCA), extractable nuclear antigen antibodies (ENA) and rheumatoid factor (RF). Serial blood and urine cultures were negative for bacterial growth.

To exclude latent infection, a computerized tomography (CT) scan of the thorax and abdomen with contrast medium was requested and revealed a dishomogeneous mass measuring $26 \times 21 \mathrm{~mm}$ with some hypodensities at the pancreatic tail, interpreted as intraductal papillary mucinous neoplasm (IPMN) which was confirmed by magnetic resonance imaging (MRI) of the abdomen.

As part of the diagnostic work-up for fever of unknown origin (FUO), a positron emission tomography/computerised tomography (PET/CT) scan was requested and revealed an increase in fluorine-18-deoxyglucose (FDG) activity in the spermatic cord (left>right) with extension to the scrotal sac (Fig. 1).
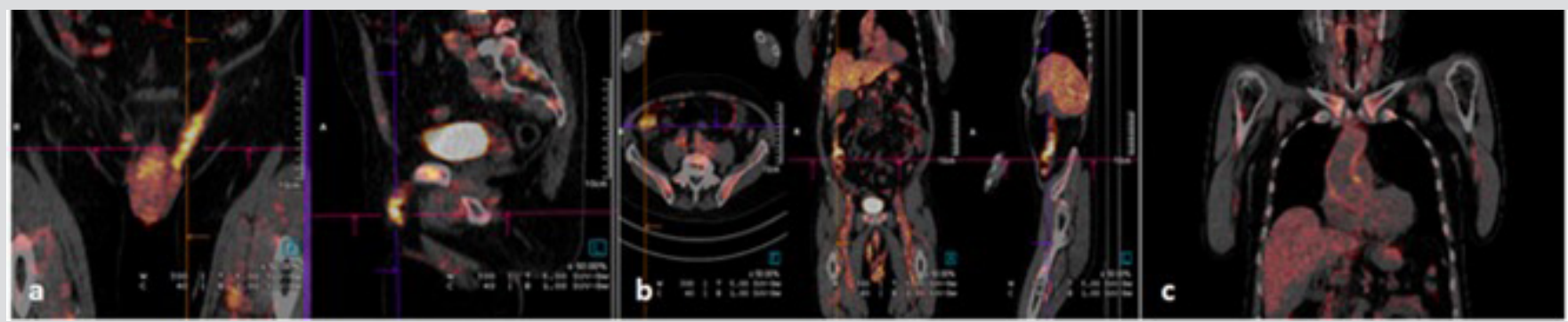

Figure 1. Positron Emission Tomography / Computed Tomography (PET / CT) shows increased activity of fluorine 18-deoxyglucose (FDG) in the spermatic cord (left> right) with extension to the scrotal sac and minimal absorption of inflammatory significance in the ascending aorta and diffuse nonspecific hyperactivity in the osteomedullary area

Furthermore, there was minimal uptake of inflammatory significance in the ascending aorta and diffuse hyperactivity in the osteomedullary area in the absence of focal localization presumed to be reactive in nature. Finally, there was intense metabolic activity in the ascending colon which was further investigated with colonoscopy that excluded significant lesions. The urological examination showed no significant findings. At this point 11 days after admission, we opted to perform a bilateral temporal artery biopsy (TAB) that showed signs of chronic inflammation of the vascular wall, compatible with giant cell arteritis (Figs. 2 and 3). The patient received high-dose steroid therapy (prednisone $1 \mathrm{mg} / \mathrm{kg} /$ daily) and is currently in complete remission.

\section{DISCUSSION}

Horton's arteritis is a systemic vasculitis of medium and large vessels with a tendency to involve the extracranial branches of the carotid arteries $^{[1]}$. This vasculitis is $2-3$ times more common in females than in males and typically occurs over the age of 50 . The mean age at the time of GCA onset is approximately 77 years, similar to that of our patient. The incidence is higher in Caucasian populations in northern Europe. The increase in incidence over the past three decades has been associated with a steady increase in TAB-positive samples ${ }^{[2]}$. Clinical features typically include headache, temporal artery tenderness, acute vision loss in 5-15\% or stroke in 3-7\% of patients, jaw claudication during chewing, polymyalgia rheumatic (PMR), or low-grade fever ${ }^{[1,3]}$. The diagnosis of GCA is based on clinical and laboratory tests and application of the 1990 ACR criteria revised in $2016^{[4]}$. TAB is still considered the gold standard for diagnosing GCA with a sensitivity of $15-40 \%$ and specificity of $100 \%$, but the temporal arteries are not involved in over a third of GCA patients ${ }^{[5]}$. Patients with clinical suspicion of GCA are referred for biopsy in a median time of 10 days ${ }^{[6]}$.

The literature suggests GCA should be considered a vasculitis with different phenotypic manifestations, where the traditional involvement of the temporal artery may be absent and the primarily involved vessels are instead the aorta or its branches, resulting in different clinical presentations. This often leads to difficulties in diagnosis because many of GCA's manifestations do not directly suggest an underlying vasculitis ${ }^{[7]}$. 


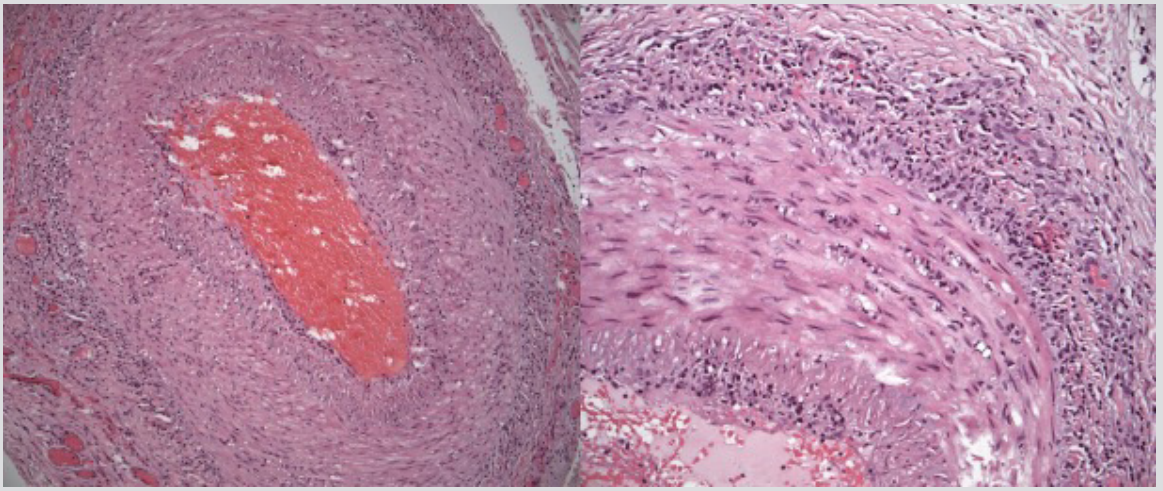

Figure 2. Histological findings. Left panel: muscular artery with partial destruction of the vessel wall by transmural lymphohistiocytic inflammatory infiltrate (HE stain ×100). Right panel: transmural lymphohistiocytic inflammatory infiltrate containing rare multinucleated giant cells (HE stain $\times 200$ )

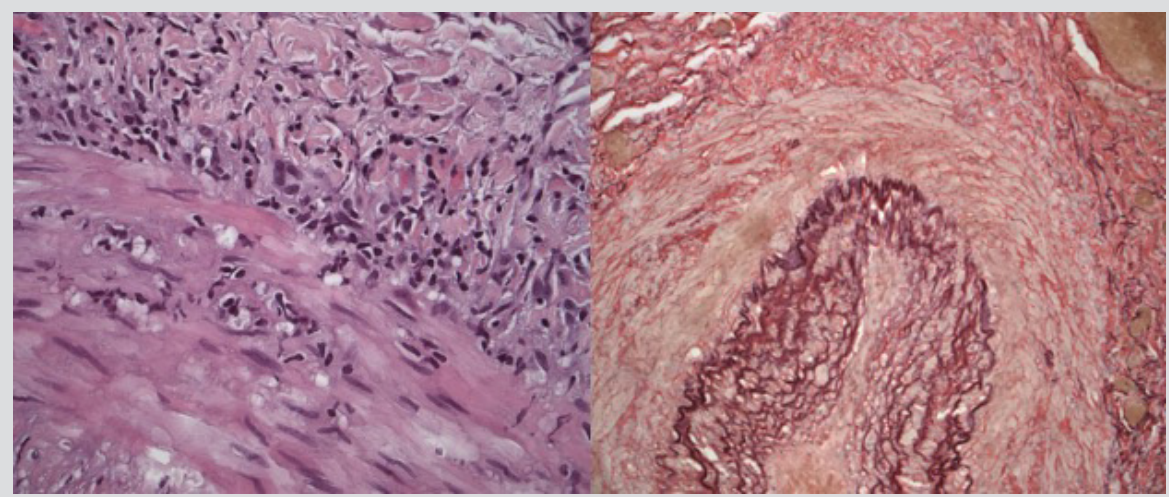

Figure 3. Histological findings. Left panel: lymphohistiocytic inflammatory infiltrate in the vessel wall (HE stain $\times 400$ ). Right panel: vessel wall with fragmentation of the elastic lamella (EvG stain ×200)

The diagnosis of GCA in patients with atypical clinical features is challenging, as demonstrated in the clinical case we have described. GCA can occur in an atypical way in up to $38 \%$ of cases, but presentation solely with fever is very rare ${ }^{[8]}$.

The most recent literature has described more than 200 possible causes of FUO. In the last decades, more cases of FUO have been due to autoimmune diseases or inflammatory processes than to infection or malignancy ${ }^{[9]}$. The first formal definition of FUO to gain broad acceptance was proposed by Petersdorf and Beeson in 1961: "fever higher than $38.3^{\circ} \mathrm{C}\left(100.9^{\circ} \mathrm{F}\right)$ on several occasions, persisting without diagnosis for at least 3 weeks in spite of at least 1 week's investigation in hospital" [10]. The definition of classic FUO was then revised by Durack as fever with an uncertain diagnosis despite 3 days in hospital or three out-patient visits ${ }^{[11]}$.

In their study, Bitik et al. report values for the differential diagnosis of increased ESR in a simple and accessible test ${ }^{[12]}$. ESR levels were elevated in diagnosed rheumatic disease or exacerbations, including CGS, with a mean value of $67.5 \mathrm{~mm} / \mathrm{h}$, while the mean ESR level was higher for infections and malignant tumours $(75 \mathrm{~mm} / \mathrm{h}$ and $85.7 \mathrm{~mm} / \mathrm{h}$, respectively).

Fluoride-18-labeled fluorodeoxyglucose (18F-FDG) positron emission tomography/computed tomography (PET/CT) has emerged in recent years for the evaluation of patients with FUO and suspected vasculitis. Some studies have shown that 18F-FDG PET/CT has high sensitivity (77-92\%) and specificity (89-100\%) in the diagnosis of vasculitis of the great vessels in patients with increased inflammatory markers, especially if the aorta or its pre-cranial branches are involved. However, this method cannot be used for the diagnosis and monitoring of inflammation localized in the temporal artery ${ }^{[13,14]}$.

The FUO work-up can be difficult when patients have atypical clinical symptoms and non-specific laboratory results. Furthermore, the course of the fever does not always follow a specific pattern.

FUO is a demanding clinical condition, and the possibility of GCA must be considered. The heterogeneity of the disorder, the lack of multicentre, high-quality studies, and the wide range of possible diagnostic techniques mean that clinical judgment remains an essential element.

Even though fever will resolve without serious complications in the many patients who remain undiagnosed despite thorough evaluation, FUO remains a difficult clinical condition and a challenge for the young physician ${ }^{[15]}$. 


\section{CONCLUSION}

The presence of isolated persistent fever (FUO) and high indices of inflammation in patients over the age of 50, should suggest Horton's arteritis even in the absence of characteristic clinical signs and symptoms. PET can be a valuable aid in the diagnosis of atypical cases, but only biopsy allows a definitive diagnosis. It is therefore essential to always consider GCA in the differential diagnosis of a patient with FUO.

\section{REFERENCES}

1. Nesher G, Breuer GS. Giant cell arteritis and polymyalgia rheumatica: 2016 update. Rambam Maimonides Med J 2016;7:e0035.

2. Gonzalez-Gay MA, Vazquez-Rodriguez TR, Lopez-Diaz MJ, Miranda-Filloy JA, Gonzalez-Juanatey C, Martin J, et al. Epidemiology of giant cell arteritis and polymyalgia rheumatica. Arthritis Rheum 2009;61(10):1454-1461

3. Kermani TA, Schafer VS, Crowson CS, Hunder GG, Gabriel SE, Matteson EL, et al. Increase in age at onset of giant cell arteritis: a population-based study. Ann Rheum Dis 2010;69:780-781.

4. Salehi-Abari I. 2016 ACR revised criteria for early diagnosis of giant cell (temporal) arteritis. Autoimmune Dis Ther Approaches Open Access 2016;3:1-4.

5. Cristaudo AT, Mizumoto R, Hendahewa R. The impact of temporal artery biopsy on surgical practice. Ann Med Surg 2016;11:47-51.

6. Sait MR, Lepore M, Kwasnicki R, Allington J, Balasubramanian R, Somasundaram SK, et al. The 2016 revised ACR criteria for diagnosis of giant cell arteritis - our case series: can this avoid unnecessary temporal artery biopsies? Int J Surg Open 2017;9:19-23.

7. Calamia KT, Hunder GG. Giant cell arteritis (temporal arteritis) presenting as fever of undetermined origin. Arthritis Rheum 1981;24:1414-1418.

8. Desmet GD, Knockaert DC, Bobbaers HJ. Temporal arteritis: the silent presentation and delay in diagnosis. J Intern Med 1990;227:237-240.

9. Naito T, Mizooka M, Mitsumoto F, Kanazawa K, Torikai K, Ohno S, et al. Diagnostic workup for fever of unknown origin: a multicenter collaborative retrospective study. BMJ Open 2013;3:e003971.

10. Petersdorf RG, Beeson PB. Fever of unexplained origin: report on 100 cases. Medicine (Baltimore) 1961;40:1-30.

11. Durack DT, Street AC. Fever of unknown origin-reexamined and redefined. Curr Clin Top Infect Dis 1991;11:35-51.

12. Bitik B, Mercan R, Tufan A, Tezcan E, Küçük H, İlhan M, et al. Differential diagnosis of elevated erythrocyte sedimentation rate and C-reactive protein levels: a rheumatology perspective. Eur J Rheumatol 2015;2:131-134.

13. Zerizer I, Tan K, Khan S, Barwick T, Marzola MC, Rubello D, et al. Role of FDG-PET and PET/CT in the diagnosis and management of vasculitis. Eur J Radiol 2010;73(3):504-509.

14. Webb M, Chambers A, AL-Nahhas A, Mason JC, Maudlin L, Rahman L, et al. The role of 18F-FDG PET in characterising disease activity in Takayasu arteritis. Eur J Nucl Med Mol Imaging 2004;31(5):627-634.

15. Wright WF, Auwaerter PG. Fever and fever of unknown origin: review, recent advances, and lingering dogma. Open Forum Infect Dis 2020;7(5):ofaa132. 